Review Article

\title{
Bcl-2 Family Proteins Are Involved in the Signal Crosstalk between Endoplasmic Reticulum Stress and Mitochondrial Dysfunction in Tumor Chemotherapy Resistance
}

\author{
Jing Su, ${ }^{1}$ Lei Zhou, ${ }^{2}$ Mei-hui Xia, ${ }^{3}$ Ye Xu, ${ }^{4}$ Xi-yan Xiang, ${ }^{1}$ and Lian-kun Sun ${ }^{1}$ \\ ${ }^{1}$ Key Laboratory of Pathobiology, Department of Pathophysiology, College of Basic Medical Sciences, Jilin University, \\ Ministry of Education, Changchun 130021, China \\ ${ }^{2}$ Department of Pathology, Affiliated Hospital to Changchun University of Chinese Medicine, Changchun 130021, China \\ ${ }^{3}$ Department of Obstetrics, First Hospital, Jilin University, Changchun 130021, China \\ ${ }^{4}$ Medical Research Laboratory, Jilin Medical College, Jilin 132013, China
}

Correspondence should be addressed to Lian-kun Sun; sunlk@jlu.edu.cn

Received 6 June 2014; Accepted 22 July 2014; Published 10 August 2014

Academic Editor: Marcos Roberto de Oliveira

Copyright (C) 2014 Jing Su et al. This is an open access article distributed under the Creative Commons Attribution License, which permits unrestricted use, distribution, and reproduction in any medium, provided the original work is properly cited.

\begin{abstract}
Tumor cells overexpress antiapoptotic proteins of the Bcl-2 (B-cell leukemia/lymphoma-2) family, which can lead to both escape from cell death and resistance to chemotherapeutic drugs. Recent studies suggest that the endoplasmic reticulum (ER) can produce proapoptotic signals, amplifying the apoptotic signaling cascade. The crosstalk between mitochondria and ER plays a decisive role in many cellular events but especially in cell death. Bcl-2 family proteins located in the ER and mitochondria can influence not only the function of the two organelles but also the interaction between them. Therefore, the Bcl-2 family of proteins may also be involved in the mechanism of tumor chemotherapy resistance by influencing crosstalk between the ER and mitochondria. In this review we will briefly discuss evidence to support this concept.
\end{abstract}

\section{Introduction}

The Bcl-2 (B-cell leukemia/lymphoma-2) family of proteins includes both antiapoptotic members (such as Bcl-2 and $\mathrm{Bcl}-\mathrm{xL}$ ) and proapoptotic members (multi-Bcl-2 homology(BH-) domain proteins such as Bax and Bak and also BH3only proteins). Bcl-2 was the first identified antiapoptotic gene and is widely expressed by hematopoietic cells, epithelial cells, lymphocytes, nerve cells, and a wide variety of tumor cells [1]. Overexpression of $\mathrm{Bcl}-2$ in tumor cells can produce tolerance to a variety of anticancer drugs and mediate escape from cell death. Based on this theory, a series of Bcl-2 inhibitors, including HA14-1, GX15-070, BI-33, ABT 737, and $\mathrm{S} 1$, were synthesized by mimicking $\mathrm{BH} 3$-only proteins to enable a breakthrough in the study of antitumor therapy [2]. These $\mathrm{BH} 3$-only protein analogs (or $\mathrm{BH} 3$ mimetics) can competitively combine with antiapoptotic proteins, including Bcl-2, Bcl-xL, Bcl-w, and Mcl-1, followed by the release of the proapoptotic proteins Bax and Bak, which ultimately induces apoptosis [3].

An increasing number of reports in the literature have confirmed that multiple organelles and genes are involved in the mechanism of tumor chemotherapy resistance [4, 5]. Two-way communication between endoplasmic reticulum (ER) and mitochondria regulates both physiological and pathological processes in cells including mitochondrial energy metabolism, lipid metabolism, $\mathrm{Ca}^{2+}$ signaling pathways, and cell death [6]. Studies have found that Bcl-2 proteins are distributed not only in the mitochondrial outer membrane, but also in the cell membrane, ER, and nuclear membrane [7]. Bcl-2 family proteins located in the ER are known to be involved in the regulation of endoplasmic reticulum signaling pathways [2]. Therefore research on the role of Bcl-2 family proteins in the delivery of proapoptotic signals to mitochondria via the ER can help us understand how tumor chemotherapy resistance develops. 
Notably, the Bcl-2 family proteins are not only involved in apoptosis, but also participate in the unfolded protein response (UPR) and calcium balance in the ER and are a focal point in the complex signaling network present in cells. In this paper, we will focus on the regulatory role of $\mathrm{Bcl}-2$ family proteins in the crosstalk between endoplasmic reticulum stress and mitochondrial dysfunction in tumor chemotherapy resistance.

\section{Bcl-2 Family Proteins Are Involved in the ER-Mediated Pathway of Apoptosis}

The ER is one of the most important membranous organelles in cells and is involved in many important cellular functions including protein folding and transportation, lipid synthesis, and maintaining calcium homeostasis [8]. The ER is the sensor for intra- and extracellular environmental stimuli and monitoring and maintaining cell homeostasis. ER provides a platform where environmental signals can connect to biological structure and function, enabling the integration of multiple stress signaling pathways [9]. The disruption of cell homeostasis will lead to ER dysfunction and gradually reduce the cell's ability to react to physiological stress. When unfolded or misfolded proteins accumulated in the ER lumina, an adaptive response called the unfolded protein response (UPR) occurs. The UPR can alleviate ER stress by reducing protein synthesis, promoting protein degradation, and producing chaperones to assist with protein folding. Excessive or prolonged ER stress can lead to cell death mediated by apoptosis [10].

Chemotherapy resistance is often due to resistance of tumor cells to cell death induced by chemotherapeutic agents, which is the result of the loss of balance between cell survival and death. Existing research work shows that antitumor agents can induce ER stress, which then activates the UPR [11]. The UPR regulates gene transcription and translation through the IRE1/XBP1 (inositol-requiring enzyme 1/X-box binding protein-1), PERK/ATF4 (PKRlike ER kinase/activating transcription factor-4), and ATF6 (activating transcription factor-6) signal pathways. The ER is considered not only an early apoptosis checkpoint to reduce cell damage, but also an important participant in the amplification of the apoptosis signaling cascade. ER stress can lead to apoptosis through the mitochondrial apoptosis pathway. The UPR plays different roles in the mechanisms of the chemotherapeutic resistance or antitumor therapy according to the stimuli, cell context, and so forth [12].

The balance between different ER-localized Bcl-2 family proteins regulates the activation of ER signaling pathways and thus cell survival. ER-localized antiapoptotic proteins such as $\mathrm{Bcl}-2$ and $\mathrm{Bcl}-\mathrm{xL}$ suppress a variety of apoptosis inducing stimuli, including ER-localized proapoptotic proteins $\mathrm{Bax} / \mathrm{Bak}$ and various $\mathrm{BH} 3$-only proteins. Both ER and mitochondrial apoptosis signaling pathways can lead to Bax and Bak activation and apoptosis, although there are differences between the two apoptosis signaling pathways [13]. Previous studies have found that under different cellular stresses, Bax and Bak can directly combine with IRE1 $\alpha$ on the cytoplasmic side of the ER membrane. IRE1 $\alpha$ then recruits ASK1, leading to activation of the proapoptotic signaling kinase JNK and downstream proapoptotic transcription factors c-Jun and p38 MAPK. This causes upregulation of CHOP/GADD153 and caspase-4 activation [14]. CHOP/GADD153 is an ER stressspecific transcription factor, which belongs to the C/EBP transcription factor family. CHOP/GADD153 plays an important role as a switch in apoptosis. ASK1 and JNK activation can also phosphorylate $\mathrm{Bcl}-2$ and the $\mathrm{BH} 3$-only protein Bim, resulting in inactivation of the antiapoptotic protein, promoting proapoptotic protein activation and induction apoptosis. ATF4 is also activated by the UPR and can directly bind the Mcl-1 promoter, regulating the transcription of $\mathrm{Mcl}-1$ [15].

Our previous work based on the $\mathrm{BH} 3$-only protein mimics S1 and ABT-737 suggests that the antitumor activities of these two small molecules are related not only to the mitochondrial apoptosis pathway, but also to the ER stressmediated pathway of apoptosis $[4,16]$. ER-localized Bcl-2 family proteins play an important role not only in regulating apoptosis induced by ER stress but also in maintaining $\mathrm{Ca}^{2+}$ homeostasis. Abnormal $\mathrm{Ca}^{2+}$ release from ER is a critical early event in apoptosis $[17,18]$. ER calcium-ATPase (SERCA) and inositol 1,4,5-trisphosphate receptor (IP3R) can regulate the processing of $\mathrm{Ca}^{2+}$ from the cytoplasm into ER and also the physiological transient release of $\mathrm{Ca}^{2+}$ back into the cytoplasm. $\mathrm{Ca}^{2+}$ release from ER during ER stress can also promote the migration of Bax to the mitochondrial membrane, resulting in Bax oligomerization in the mitochondrial outer membrane and cytochrome c [19]. Bcl-2 and Bcl$\mathrm{xL}$ can affect IP3R in a variety of ways, thus reducing the endoplasmic reticulum $\mathrm{Ca}^{2+}$ capacity, leading to decreases in cytoplasmic $\mathrm{Ca}^{2+}$ and the mitochondrial $\mathrm{Ca}^{2+}$ response, thus inhibiting $\mathrm{Ca}^{2+}$-dependent cell death [20]. Furthermore, knockout of the proapoptotic genes Bax and Bak can also lead to a reduction in the endoplasmic reticulum $\mathrm{Ca}^{2+}$ capacity [21]. Thus, Bcl-2 family proteins can influence apoptosis through the regulation of intracellular $\mathrm{Ca}^{2+}$ homeostasis.

\section{The Bcl-2 Family of Proteins Regulates Contact between ER and Mitochondria}

ER and mitochondria are closely connected, both structurally and functionally. Recent studies have further confirmed that the connection between ER and mitochondria (endoplasmic reticulum-mitochondria contacts) is the foundation of cell organelle communication [22]. This two-way communication is involved in the regulation of a variety of physiological and pathological processes, including mitochondrial energy metabolism, lipid metabolism, $\mathrm{Ca}^{2+}$ signaling ER pathways, and cell death [23]. Mitochondria are connected to the endoplasmic reticulum at specific sites called mitochondrionassociated membranes (MAMs). MAMs are adjacent to the $\mathrm{ER}$ and contain important $\mathrm{Ca}^{2+}$ transport-related proteins including IP3R, VDAC, GRP75, Sig-1R, and Bip [24]. The changing of morphology, intracellular ATP, or $\mathrm{Ca}^{2+}$ concentration can all affect ER-mitochondria contacts; therefore all 
of these factors may also be involved in regulating two-way communication between mitochondria and ER.

Bcl-2 family proteins are involved in the regulation of mitochondrial fission and fusion and can also control the morphology of mitochondria, thus affecting signaling between mitochondria and ER. Recent studies found that mitochondria form a reticular structure similar to ER in most kinds of cells. Mitochondrial reticular structure undergoes dynamic changes due to the processes of mitochondrial fission and fusion, which are precisely controlled by a variety of proteins. Drpl, Fis1, Caf4p, and Mdvlp are involved in the regulation of mitochondrial division. Mfn1/2 and OPA1 control the fusion of mitochondrial outer or inner membranes, respectively [25]. The Bcl-2 homolog CED-9 can interact with the mitochondria fusion protein Mfn2 and induce mitochondrial fusion in C. elegans. Bcl-xL can inhibit the GTPase activity of the mitochondrial fission protein Drp1. In contrast, the proapoptotic proteins Bak and Bax promote mitochondrial fission mediated by Drp1 [26]. Overexpression of the Bcl-2 family proapoptotic protein Bax can promote Drpl-dependent mitochondrial fragmentation [27]. Bax and Bak promote mitochondrial fragmentation by stabilizing the interaction of Drp1 and mitochondria during apoptosis [28]. Studies have shown that Noxa and Puma also promote Drp1dependent mitochondrial fragmentation [13]. In summary, Bcl-2 family proteins can affect the balance of mitochondrial fission/fusion and thus regulate the morphology and function of mitochondria.

Bcl-2 family proteins can indirectly affect ER-mitochondria contacts by interacting with mitochondrial fission/fusion proteins as outlined above. Studies have shown that Mfn2 is distributed not only in mitochondria, but also in the ER surface linked to mitochondria. At the particular sites where mitochondria are connected with ER, the mitochondrial outer membrane fusion proteins Mfnl and Mfn2 can form homo- or heterodimers in the ER membrane [14]. In Mfn2-defficient fibroblasts, the distance between ER and mitochondria increased, and the morphology of both organelles is changed. Mitochondrial fusion proteins are essential in both mitochondria and ER to connect the two organelles [15]. Additionally, the capacity of mitochondria for uptake of $\mathrm{Ca}^{2+}$ decreases after $\mathrm{ER} \mathrm{Ca}^{2+}$ release induced by inositol triphosphate (IP3) in Mfn2-deficient cells. This indicates that Mfn2 plays an important role in maintaining $\mathrm{Ca}^{2+}$ signaling between mitochondria and ER and also in the $\mathrm{Ca}^{2+}$ capacity of mitochondria $[15,29]$. According to a new study, the crosstalk between ER and mitochondria in apoptosis is mediated by the mitochondrial fission proteins Drp1 and Fis1. Fis1 can form a complex with the ER protein Bap31, which is mainly located where ER is connected with mitochondria. Fis1-Bap31 compounds can provide a platform for recruiting and activating caspase-8 early in apoptosis. Bap31 is then cleaved, leading to increased $\mathrm{Ca}^{2+}$ release from ER and induction of cytochrome $c$ release from mitochondria [30]. In addition to Bap31 cleavage, Bik located in ER has also been shown to cause apoptosis mediated by $\mathrm{Ca}^{2+}$, leading to Drpl-dependent mitochondrial fission and the release of cytochrome c [22, 31].

\section{The Bcl-2 Family Proteins Involved in Autophagy}

Recent research confirmed that Bcl-2 family proteins including $\mathrm{Bcl}-2$, Bax, Bak, and Bik are not only involved in the regulation of apoptosis but also regulate autophagy, which plays an important role in cell survival and death. Autophagy is a highly conserved strictly regulated process involving the degradation of long-life proteins and damaged organelles. Autophagy promotes cell survival under various stress conditions including ER stress or oxidative stress [32]. Studies have shown that autophagy is an important part of the normal function of the ER $[33,34]$. ER stress-induced autophagy plays a central role in alleviating stress and maintaining homeostasis. There are two main cellular degradation systems present in eukaryotic cells: the ubiquitin-proteasome and autophagy-lysosome systems. The ER is associated with both degradation processes. Autophagy induced by ER stress is an alternative process of ER-associated protein degradation (ERAD) to degradation of misfolded proteins in the ER lumen. Autophagy can also degrade damaged ER itself to maintain ER function and homeostasis.

The loss of mitochondrial membrane potential can lead to both the release of proapoptotic factors from mitochondria and the production of reactive oxygen species. Dysfunctional mitochondria will be selectively cleared by autophagy. The healthy mitochondria are mainly through the PINK1mediated phosphorylation of kinases reaction and subsequent E3 ligase Parkin-mediated ubiquitin reaction to clear them [10].

Studies have found that the $\mathrm{BH} 3$-only protein mimics ABT-737 and HA14-1 can competitively inhibit ER-localized $\mathrm{Bcl}-2$ and $\mathrm{Bcl}-\mathrm{xL}$ and prevent $\mathrm{Bcl}-2 / \mathrm{Bcl}-\mathrm{xL}$ combining with Beclinl and the activation of Beclinl-dependent autophagy [35-37]. Moreover, $\mathrm{BH} 3$-only protein mimics can also regulate cell autophagy by other pathways [38]. Activation of the UPR signaling pathway PERK-eIF2 $\alpha$-ATF4 can upregulate the expression of the autophagy gene Atg12 [39]. The interaction of IRE $\alpha$ and Bax/Bak can also promote the activation of the JNK pathway during ER stress. Notably, the early period of JNK activation promotes autophagy, while continuous activation of JNK leads to apoptosis [40, 41].

$\mathrm{S} 1$ is a small-molecule mimic of the $\mathrm{BH} 3$-only protein Bid [42]. Studies based on two-dimensional nuclear magnetic and fluorescence polarization experiments show that S1 has a high affinity for $\mathrm{Bcl}-2$ and $\mathrm{Mcl}-1$. S1 can therefore interfere with the interactions of Bcl-2/Bax or Mcl-1/Bak, thus inducing apoptosis in various tumor cells [43], including liver and breast cancer [44]. Our recent study shows that S1 can also induce apoptosis in glioma U251 cells, accompanied by upregulation of the expression of the autophagy marker protein LC3-II and ER stress marker protein PDI. This indicates that ER stress and autophagy are involved in the antitumor effects of S1, in addition to apoptosis. Our research work suggests that S1 can also induce ER stress-mediated apoptosis $[4,16]$. 


\section{Conclusion}

The findings detailed above demonstrate that Bcl-2 family proteins are a common link between ER stress, mitochondrial dysfunction, and autophagy. Further studies are required to confirm the regulatory role of $\mathrm{Bcl}-2$ family proteins in the crosstalk between endoplasmic reticulum stress and mitochondrial dysfunction in tumor chemotherapy resistance. However, these data provide hope for the development of novel antitumor therapeutic strategies based on Bcl-2 inhibitors to overcome tumor chemotherapy resistance.

\section{Conflict of Interests}

The authors declare that they have no conflict of interests regarding the publication of this paper.

\section{Acknowledgments}

This research was funded by the National Natural Science Foundation of China (nos. 81272876, 81202552, and 81372793) and " 211 Project" of Jilin University.

\section{References}

[1] R. J. Youle and A. Strasser, "The BCL-2 protein family: opposing activities that mediate cell death," Nature Reviews Molecular Cell Biology, vol. 9, no. 1, pp. 47-59, 2008.

[2] S. Cory and J. M. Adams, "The BCL2 family: regulators of the cellular life-or-death switch," Nature Reviews Cancer, vol. 2, no. 9, pp. 647-656, 2002.

[3] M. H. Kang and C. P. Reynolds, "BcI-2 Inhibitors: targeting mitochondrial apoptotic pathways in cancer therapy," Clinical Cancer Research, vol. 15, no. 4, pp. 1126-1132, 2009.

[4] N. Liu, Y. Xu, J. T. Sun et al., "The BH3 mimetic S1 induces endoplasmic reticulum stress-associated apoptosis in cisplatinresistant human ovarian cancer cells although it activates autophagy," Oncology Reports, vol. 30, no. 6, pp. 2677-2684, 2013.

[5] H. Yu, J. Su, Y. Xu et al., "P62/SQSTM1 involved in cisplatin resistance in human ovarian cancer cells by clearing ubiquitinated proteins," European Journal of Cancer, vol. 47, no. 10, pp. 1585-1594, 2011.

[6] J. D. Malhotra and R. J. Kaufman, "ER stress and its functional link to mitochondria: role in cell survival and death," Cold Spring Harbor Perspectives in Biology, vol. 3, no. 9, 2011.

[7] S. Krajewski, S. Tanaka, S. Takayama, M. J. Schibler, W. Fenton, and J. C. Reed, "Investigation of the subcellular distribution of the bcl-2 oncoprotein: residence in the nuclear envelope, endoplasmic reticulum, and outer mitochondrial membranes," Cancer Research, vol. 53, no. 18, pp. 4701-4714, 1993.

[8] M. J. Berridge, "The endoplasmic reticulum: a multifunctional signaling organelle," Cell Calcium, vol. 32, no. 5-6, pp. 235-249, 2002.

[9] C. Giorgi, D. De Stefani, A. Bononi, R. Rizzuto, and P. Pinton, "Structural and functional link between the mitochondrial network and the endoplasmic reticulum," International Journal of Biochemistry and Cell Biology, vol. 41, no. 10, pp. 1817-1827, 2009.
[10] J. Su, L. Zhou, X. Kong et al., "Endoplasmic reticulum is at the crossroads of autophagy, inflammation, and apoptosis signaling pathways and participates in the pathogenesis of diabetes mellitus," Journal of Diabetes Research, vol. 2013, Article ID 193461, 6 pages, 2013.

[11] T. Verfaillie, A. D. Garg, and P. Agostinis, “Targeting ER stress induced apoptosis and inflammation in cancer," Cancer Letters, vol. 332, no. 2, pp. 249-264, 2013.

[12] M. Moenner, O. Pluquet, M. Bouchecareilh, and E. Chevet, "Integrated endoplasmic reticulum stress responses in cancer," Cancer Research, vol. 67, no. 22, pp. 10631-10634, 2007.

[13] H. Woo, Y. Seo, A. R. Moon, S. Jeong, E. K. Choi, and T. Kim, "Effects of the BH3-only protein human Noxa on mitochondrial dynamics," The FEBS Letters, vol. 583, no. 14, pp. 2349-2354, 2009.

[14] O. M. de Brito and L. Scorrano, "An intimate liaison: spatial organization of the endoplasmic reticulum-mitochondria relationship," The EMBO Journal, vol. 29, no. 16, pp. 2715-2723, 2010.

[15] O. M. de Brito and L. Scorrano, "Mitofusin-2 regulates mitochondrial and endoplasmic reticulum morphology and tethering: the role of Ras," Mitochondrion, vol. 9, no. 3, pp. 222-226, 2009.

[16] J. T. Zhong, Y. Xu, H. Yi et al., “The BH3 mimetic S1 induces autophagy through ER stress and disruption of Bcl-2/Beclin 1 interaction in human glioma U251 cells," Cancer Letters, vol. 323, no. 2, pp. 180-187, 2012.

[17] A. W. Jones and G. Szabadkai, " $\mathrm{Ca}^{2+}$ transfer from the ER to mitochondria: channeling cell death by a tumor suppressor," Developmental Cell, vol. 19, no. 6, pp. 789-790, 2010.

[18] R. Rizzuto, S. Marchi, M. Bonora et al., " $\mathrm{Ca}^{2+}$ transfer from the ER to mitochondria: when, how and why," Biochimica et Biophysica Acta, vol. 1787, no. 11, pp. 1342-1351, 2009.

[19] P. Pinton, C. Giorgi, R. Siviero, E. Zecchini, and R. Rizzuto, "Calcium and apoptosis: ER-mitochondria Ca2+ transfer in the control of apoptosis," Oncogene, vol. 27, no. 50, pp. 6407-6418, 2008.

[20] P. Pinton, D. Ferrari, E. Rapizzi, F. di Virgilio, T. Pozzan, and R. Rizzuto, "The $\mathrm{Ca}^{2+}$ concentration of the endoplasmic reticulum is a key determinant of ceramide-induced apoptosis: significance for the molecular mechanism of Bcl-2 action," The EMBO Journal, vol. 20, no. 11, pp. 2690-2701, 2001.

[21] L. Scorrano, S. A. Oakes, J. T. Opferman et al., "BAX and BAK regulation of endoplasmic reticulum $\mathrm{Ca}^{2+}$ : a control point for apoptosis," Science, vol. 300, no. 5616, pp. 135-139, 2003.

[22] M. Germain, J. P. Mathai, H. M. McBride, and G. C. Shore, "Endoplasmic reticulum BIK initiates DRP1-regulated remodelling of mitochondrial cristae during apoptosis," EMBO Journal, vol. 24, no. 8, pp. 1546-1556, 2005.

[23] S. Grimm, "The ER-mitochondria interface: the social network of cell death," Biochimica et Biophysica Acta-Molecular Cell Research, vol. 1823, no. 2, pp. 327-334, 2012.

[24] A. Parekh, "Calcium signalling: mitofusins promote interorganellar crosstalk," Current Biology, vol. 19, no. 5, pp. R200R203, 2009.

[25] R. J. Youle and A. M. Van der Bliek, "Mitochondrial fission, fusion, and stress," Science, vol. 337, no. 6098, pp. 1062-1065, 2012.

[26] R. Jagasia, P. Grote, B. Westermann, and B. Conradt, "DRP-1mediated mitochondrial fragmentation during EGL-1-induced cell death in C. elegans," Nature, vol. 433, no. 7027, pp. 754-760, 2005. 
[27] S. Frank, B. Gaume, E. S. Bergmann-Leitner et al., "The Role of Dynamin-Related Protein 1, a Mediator of Mitochondrial Fission, in Apoptosis," Developmental Cell, vol. 1, no. 4, pp. 515525,2001

[28] S. Wasiak, R. Zunino, and H. M. McBride, "Bax/Bak promote sumoylation of DRP1 and its stable association with mitochondria during apoptotic cell death," Journal of Cell Biology, vol. 177, no. 3, pp. 439-450, 2007.

[29] O. M. de Brito and L. Scorrano, "Mitofusin 2 tethers endoplasmic reticulum to mitochondria," Nature, vol. 456, no. 7222, pp. 605-610, 2008.

[30] R. Iwasawa, A. Mahul-Mellier, C. Datler, E. Pazarentzos, and S. Grimm, "Fis1 and Bap31 bridge the mitochondria-ER interface to establish a platform for apoptosis induction," EMBO Journal, vol. 30, no. 3, pp. 556-568, 2011.

[31] J. P. Mathai, M. Germain, and G. C. Shore, "BH3-only BIK regulates BAX,BAK-dependent release of $\mathrm{Ca}^{2+}$ from endoplasmic reticulum stores and mitochondrial apoptosis during stressinduced cell death," The Journal of Biological Chemistry, vol. 280, no. 25, pp. 23829-23836, 2005.

[32] T. Yorimitsu and D. J. Klionsky, "Autophagy: molecular machinery for self-eating," Cell Death and Differentiation, vol. 12, supplement 2, pp. 1542-1552, 2005.

[33] M. Ogata, S. Hino, A. Saito et al., "Autophagy is activated for cell survival after endoplasmic reticulum stress," Molecular and Cellular Biology, vol. 26, no. 24, pp. 9220-9231, 2006.

[34] Y. Kouroku, E. Fujita, I. Tanida et al., "ER stress (PERK/eIF2 $\alpha$ phosphorylation) mediates the polyglutamine-induced LC3 conversion, an essential step for autophagy formation," Cell Death and Differentiation, vol. 14, no. 2, pp. 230-239, 2007.

[35] E. Szegezdi, D. C. MacDonald, T. N. Chonghaile, S. Gupta, and A. Samali, "Bcl-2 family on guard at the ER," American Journal of Physiology-Cell Physiology, vol. 296, no. 5, pp. C941-C953, 2009.

[36] L. W. Thomas, C. Lam, and S. W. Edwards, "Mcl-1; the molecular regulation of protein function," FEBS Letters, vol. 584, no. 14, pp. 2981-2989, 2010.

[37] M. Germain and R. S. Slack, "MCL-1 regulates the balance between autophagy and apoptosis," Autophagy, vol. 7, no. 5, pp. 549-551, 2011.

[38] S. A. Malik, S. Shen, G. Mariño, A. BenYounès, M. C. Maiuri, and G. Kroemer, "BH3 mimetics reveal the network properties of autophagy-regulatory signaling cascades," Autophagy, vol. 7, no. 8, pp. 914-916, 2011.

[39] G. Velasco, T. Verfaillie, M. Salazar, and P. Agostinis, "Linking ER stress to autophagy: potential implications for cancer therapy," International Journal of Cell Biology, vol. 2010, Article ID 930509, 19 pages, 2010.

[40] G. Verma and M. Datta, "The critical role of JNK in the ERmitochondrial crosstalk during apoptotic cell death," Journal of Cellular Physiology, vol. 227, no. 5, pp. 1791-1795, 2012.

[41] P. Walter and D. Ron, "The unfolded protein response: from stress pathway to homeostatic regulation," Science, vol. 334, no. 6059, pp. 1081-1086, 2011.

[42] Z. Zhang, G. Wu, J. Gao, and T. Song, "Inclusion complex of a Bcl-2 inhibitor with cyclodextrin: characterization, cellular accumulation, and in vivo antitumor activity," Molecular Pharmaceutics, vol. 7, no. 4, pp. 1349-1354, 2010.

[43] Z. Zhang, T. Song, T. Zhang et al., "A novel BH3 mimetic S1 potently induces Bax/Bak-dependent apoptosis by targeting both Bcl-2 and Mcl-1," International Journal of Cancer, vol. 128, no. 7, pp. 1724-1735, 2011.
[44] T. Song, Q. Chen, X. Li, G. Chai, and Z. Zhang, "Correction to "3-Thiomorpholin-8-oxo-8H-acenaphtho[1,2-b]pyrrole-9carbonitrile (S1) based molecules as potent, dual inhibitors of B-cell lymphoma 2 (Bcl-2) and myeloid cell leukemia sequence 1 (Mcl-1): structure-based design and structureactivity relationship studies',' Journal of Medicinal Chemistry, vol. 56, no. 22, pp. 9366-9367, 2013. 

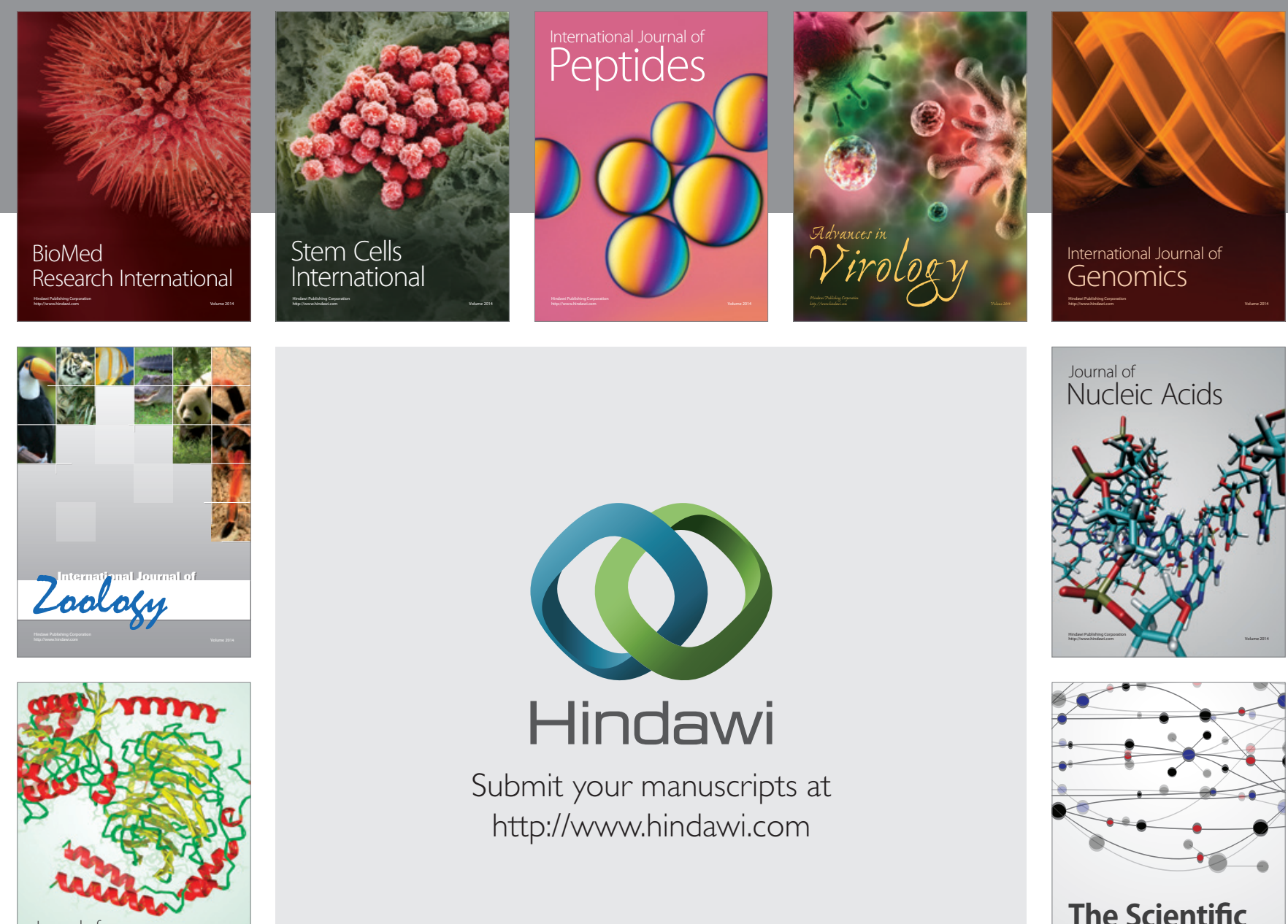

Submit your manuscripts at

http://www.hindawi.com

Journal of
Signal Transduction
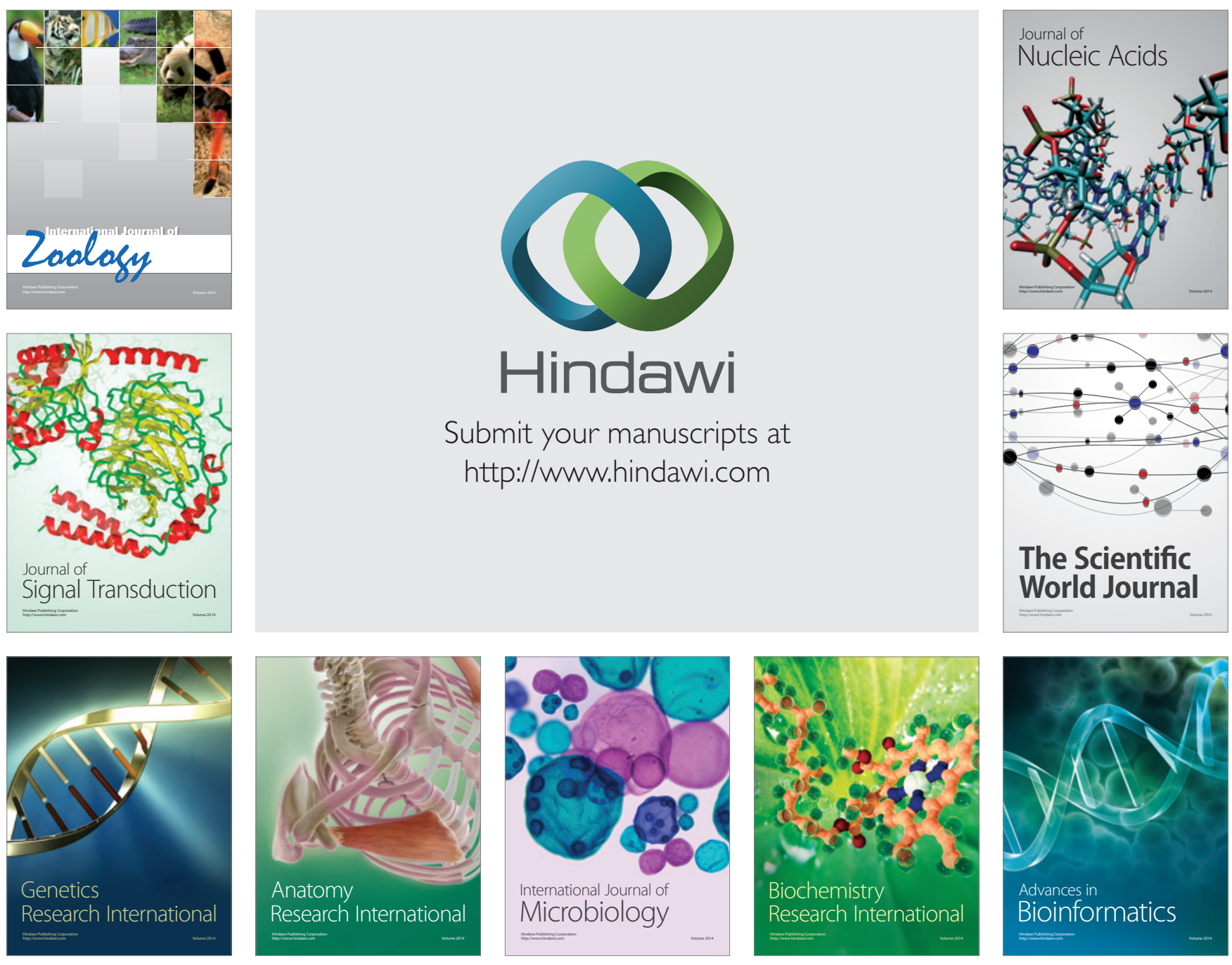

The Scientific World Journal
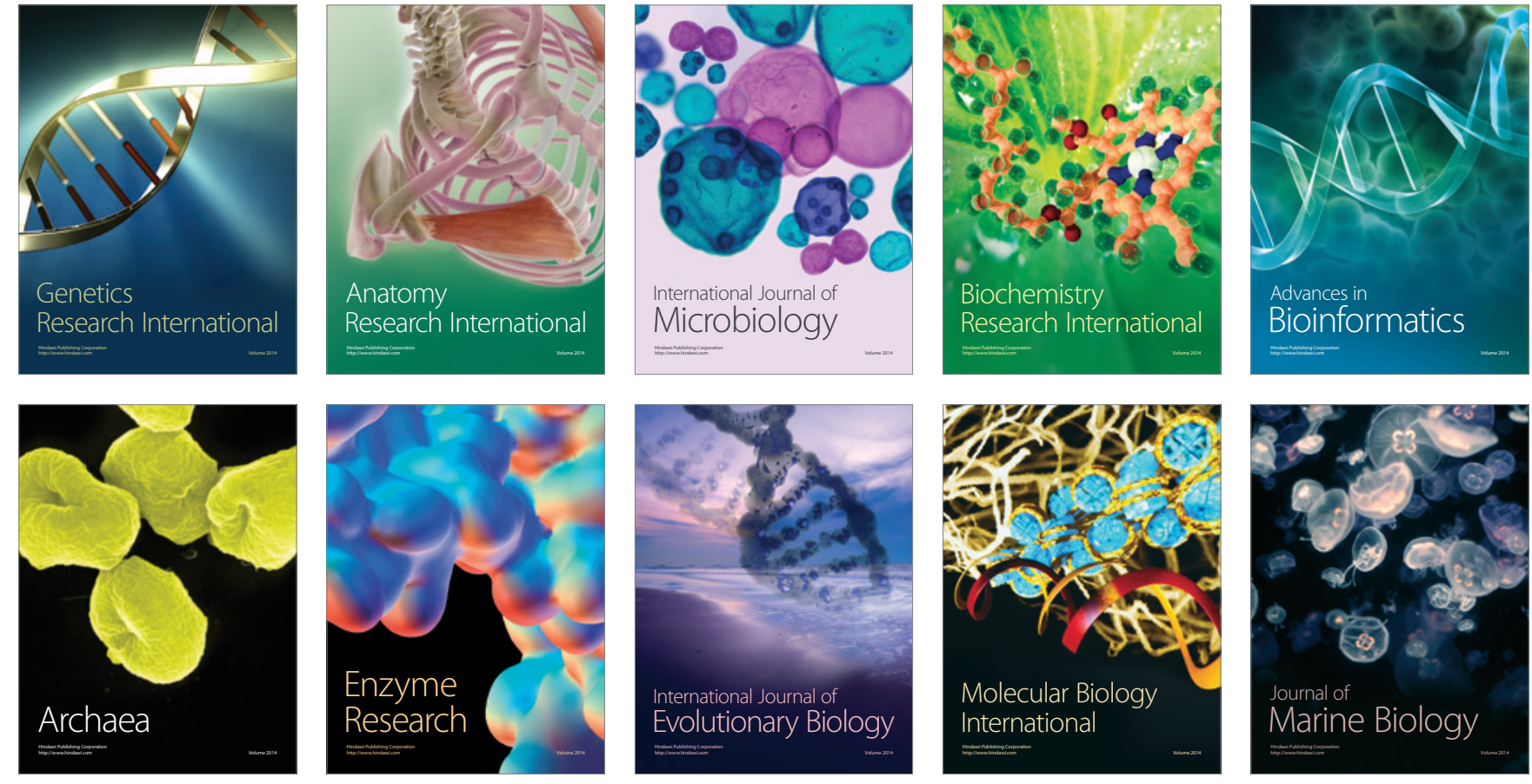